\title{
EDITORIAL
}

\section{Spotlight on molecular targeted therapy: introduction}

\author{
MS Sheikh \\ Department of Pharmacology, SUNY Upstate Medical University, Syracuse, NY, USA
}

\section{Leukemia (2002) 16, 431-432. DOI: 10.1038/sj/leu/2402418}

Recent advances in genetics, biochemistry, cell and molecular biology have greatly improved our understanding of the molecular mechanisms of neoplastic transformation and progression. There are now significant research opportunities to identify and exploit molecular targets for the discovery of anticancer agents. It is now becoming possible to target molecules and/or pathways that are crucial in maintaining the malignant phenotype. A case in point is the recent success of the therapeutic STI-571 (Gleevec) in chronic myeloid leukemia $(\mathrm{CML})$, which targets Bcr-Abl. The initial success of STI571 in clinical trials is an indication that we have already started to reap the fruit of modern biomedical research. However, unlike the Bcr-Abl-dependent CML where the target is rather obvious, other malignancies appear to harbor many abnormalities and hence, multiple potential targets. Given that the field of 'Molecular Targeted Therapy' is still evolving, much needs to be done to identify the existing and/or novel molecules and/or pathways that could be exploited as molecular targets in the development of newer, less toxic and more efficacious anticancer agents.

Starting in this issue of Leukemia, the 'Spotlight' forum presents, 'Spotlight on Molecular Targeted Therapy'. This 'Spotlight' will feature several review articles on different topics from this emerging area of research. The contributing authors have highlighted the key molecules and/or pathways that could potentially serve as important targets to develop novel anticancer agents. They have also presented their vision as to where the future of this field is heading.

Several review articles are being featured in this 'Spotlight'. The review article by Drs Jason B Almond and Gerald M Cohen discusses the ubiquitin-proteasome system as a possible target for anticancer agents. In particular, the authors discuss the utility of proteasome inhibitors as potential anticancer agents. Thus, the identification and use of novel agents to exploit the ubiquitin-proteasome to modulate the function of other molecules is believed to be of considerable benefit in achieving the goal of anticancer therapy. Dr Ruth Craig in her article describes the importance of Bcl-2 family members in the maintenance of tissue homeostasis and function. She also discusses the utility of Bcl-2 and its family member MCL1 as potential targets to develop novel anticancer agents. In his article, Dr Mikhail V Blagosklonny reviews Hsp-90 targeting by geldanamycin and its analogs. Geldanamycin and its analogs were initially thought to mediate their effects by non-specific inhibition of tyrosine and serine/thereonine kinases. However, several lines of recent evidence suggest that these

Correspondence: MS Sheikh, SUNY Upstate Medical University, Department of Pharmacology, 750 E Adams St, Syracuse, NY 13210, USA

Received 1 December 2001; accepted 14 December 2001 compounds mediate their effects by targeting Hsp-90. The anticancer potential of the allyl amino derivative of geldanamycin is currently being evaluated in phase I clinical trials. Drs Joseph A Fontana and Arun K Rishi review the molecular mechanisms of classical and novel retinoids in context to cancer therapy. They discuss, in detail, the synthetic retinoids 4HPR and CD437 and their potential therapeutic efficacies in the treatment of various malignant diseases. Several molecules serving as potential molecular targets of these retinoids are also listed in their article.

Newer and state-of-the-art tools are needed in the discovery and validation of molecular targets. In their article, Drs Michael S Orr and Uwe Scherf describe the utility and progress of gene chip microarray approaches in the discovery and validation of molecular drug targets. They also highlight the difficulties that still exist in the pursuit of such goals. Unlike DNA microarray analyses, expression proteomics seeks to explore global alterations in protein expression. Significant efforts and resources have been invested by academia and the pharmaceutical industry in the development and application of proteomics tools. In the article by Dr Samir M Hanash and colleagues, the emerging field of proteomics and its utility in discovering molecular targets have been discussed.

The article by Drs John T Lee Jr and James A McCubrey focuses on the Raf/MEK/ERK signaling cascade. In particular, the authors discuss different approaches to target various components of this potentially important signaling cascade for therapeutic interventions. Dr Linda Z Penn and colleagues describe statin family of drugs in their article. Statins inhibit 3hydroxy-3-methylglutaryl coenzyme A (HMG-CoA) reductase and have been reported to exhibit higher tumoricidal activity than the non-transformed cells. Statins appear to mediate their anticancer effects by inducing apoptosis and their apoptotic effects occur, at least in part, via cellular depletion of geranylgeranyl pyrophosphate (GGPP). Additional in-depth studies are, however, needed to further illuminate the exact molecular mechanisms by which statins mediate their anticancer effects (an original article by Dr AC Bloem and colleagues describing lovastatin-induced apoptosis in myeloma plasma cells will be published in a subsequent issue). In their article, Drs Manish Monga and Edward A Sausville give an overview of the 'Developmental Therapeutics Program at the National Cancer Institute, USA' and its role in molecular target and drug discovery. Their article also lists various reagents and free services available to the extramural scientific community interested in molecular target and drug discovery. Myeloid Differentiation (MyD) and Growth Arrest and DNA Damageinducible (GADD) genes encode a group of proteins that are involved in differentiation and cellular stress response. In their article, Drs Dan A Liebermann and Barbara Hoffman discuss the MyD/GADD gene products as possible targets for cancer therapy.

This Spotlight will continue to feature review articles on different and important topics from this emerging area of 
research. All readers are invited to contribute an article pertinent to this Spotlight. The articles published in Spotlights are always peer-reviewed. Interested parties should contact the Leukemia Editorial Office for more information on how to participate in 'Spotlight on Molecular Targeted Therapy'.

I am grateful to Dr Muller-Bérat for her insightful advice, excellent support and cooperation. 